\title{
Redundant Feature Identification and Redundancy Analysis for Causal Feature Selection
}

\author{
Asavaron Limshuebchuey \\ Department of Electrical Engineering, Faculty of Engineering \\ Prince of Songkla University, Hat Yai, Songkhla, 90112, \\ Thailand \\ 5710120062@email.psu.ac.th
}

\author{
Rakkrit Duangsoithong \\ Department of Electrical Engineering, Faculty of Engineering \\ Prince of Songkla University, Hat Yai, Songkhla, 90112, \\ Thailand \\ rakkrit.d@psu.ac.th
}

Terry Windeatt

Centre for Vision, Speech and Signal Processing

University of Surrey, UK

t.windeatt@surrey.ac.uk

\begin{abstract}
High dimensional data can lead to low accuracy of classification and take a long time to calculate because it contains irrelevant features and redundant features. To overcome this problem, dimension of data has to be reduced. Causal feature selection is one of methods for feature reduction but it cannot identify redundant features. This paper presents Parent-Children based for Causal Redundant Feature Identification (PCRF) algorithm to identify and remove redundant features. The accuracy of classification and number of feature reduced by PCRF algorithm are compared with correlation feature selection. According to the results, PCRF algorithm can identify redundant feature but has lower accuracy of classification than correlation feature selection.
\end{abstract}

Keywords- feature selection, correlation, causal feature selection, irrelevant feature, redundant feature.

\section{INTRODUCTION}

Currently, information technology is rapidly advancement that increases number of features in data. Analysis of high dimensional data often has low accuracy of classification and high computation time because curse of dimensionality [1] and over-fitting problem. It is a result of dataset that has irrelevant and redundant features. Therefore, number of features should be reduced to increase the accuracy of classification.

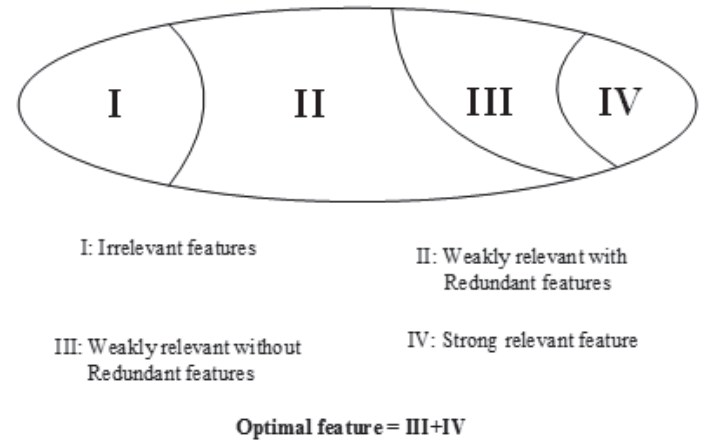

Fig. 1. Component of feature of dataset

Basically, component of feature in dataset has four parts [2] as shown in figure 1; Irrelevant features, relevant with redundant features, relevant without redundant features and strongly relevant features. The optimal feature is a dataset without irrelevant and redundant features as shown in III and IV of component in figure 1 .

Normally, feature reduction has two methods [3]; feature extraction and feature selection. Feature extraction transforms original dataset to lower dimension of data and changes value of original dataset. Feature selection chooses the optimal feature set by measuring relation such as mutual information, distance, statistics, etc. It can find relation between features such as diagnosis or causes of disease analysis. Therefore, this research focuses on feature selection because it is easy to understand and can identify optimal feature.

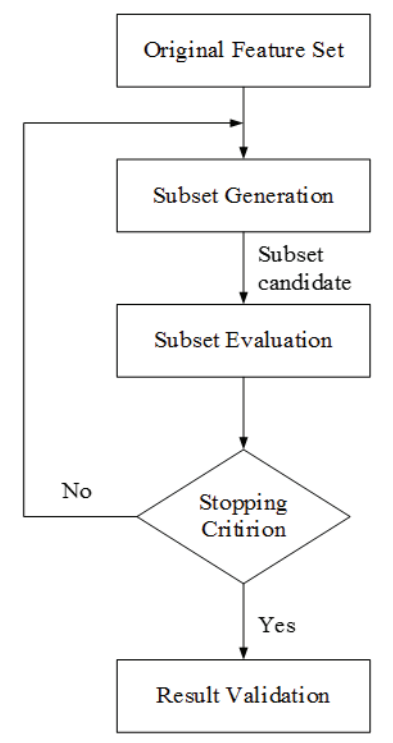

Fig. 2. Feature selection procedure

Generally, feature selection consists of four processes [4] as shown in figure 2. Subset generation is a process of search strategy such as complete search and random search. Complete search is a method that all of feature in dataset is evaluated. It

978-1-4673-9158-0/15/\$31.00 C2015 IEEE 
guarantees that final process can find the optimal feature but take a long time for searching algorithm. Random search is a method that uses random condition to find features by condition. It has rapid process but some relevant features might not be chosen. After that subset evaluation will evaluate features from the first process using criterion such as mutual information, distance, dependency, etc. This process will be iterated until reach stopping conditional criterion in stopping criterion process such as completed search or maximum number of iteration. Finally subset validation will separate optimal feature to test the optimal feature.

Feature selection algorithm can be divided into five types [5], [6]; Filter method selects feature that is not independent with the class. It is a rapid method but selected features might not be suitable for selected classification. Wrapper method defines the evaluation method that is suitable for classification to evaluate features. It has high accuracy and high computation time. Hybrid method combines advantage of filter method and wrapper method. This method has higher accuracy than filter method and faster than wrapper method. Embedded method is a method that subset evaluation is a part of classification algorithm. Markov blanket method can find the relation between class or target and features based on statistics.

Basically, feature selection does not consider causality between features but causal feature selection [7] can explain relation of all features in dataset by causal graph as shown in figure 3. Causal feature selection uses Bayesian network to calculate relation of all feature which consist of nodes (features), direct acyclic graph (DAG) and joint probability distribution between features [8].

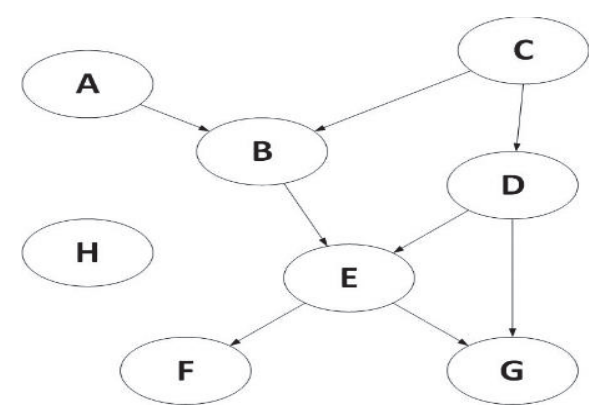

Fig. 3. Causal feature selection

\section{LITERATURE REVIEW}

Reducing the number of feature to get optimal size can increase the accuracy of classification and reduce computation time. In 1999, Hall. [9] reduced number of feature using CFS algorithm that can remove irrelevant features but cannot identify and eliminate redundant features. In 2003, Yu and Liu [10] used FCBF algorithm to reduce feature in high dimensional data that can identify both irrelevant features and redundant features by symmetrical uncertainty calculation. mRMR algorithm was presented by Peng et al. [11] in 2005. It can find optimal features and reduce number of features but cannot identify irrelevant features and redundant features from removed features.
Although these algorithm can identify optimal features, they do not consider the causality of features.

In 2000, Spirtes et al. [12] found that Bayesian network is applied to reduce features in PC algorithm but cannot identify and remove redundant features. Therefore, this paper proposes redundant feature identification and redundancy analysis for causal feature selection by Parent-Children based for Causal Redundant Feature Identification (PCRF) algorithm and compares the result of accuracy of classification and the number of feature reduction with correlation feature selection.

\section{THEORY}

This paper proposes Parent-Children based for Causal Redundant Feature Identification (PCRF) algorithm. Number of feature reduction and accuracy of classification are compared with correlation method (CFS, FCBF, mRMR algorithm) and causal feature selection (PC algorithm).

\section{A. Correlation Feature Selection}

1) Correlation based Feature Selection (CFS) Algorithm

CFS algorithm [9] measures relation between feature and feature and feature and class in equation 1 and uses Pearson's correlation in equation 2 .

$$
\begin{gathered}
\text { Merit }_{s}=\frac{k r_{k c}}{\sqrt{k+(k-1) r_{k k}}} \\
r=\frac{\sum_{i}\left(x_{i}-\bar{x}_{i}\right)\left(y_{i}-\bar{y}_{i}\right)}{\sqrt{\sum_{i}\left(x_{i}-\bar{x}_{i}\right)^{2}} \sqrt{\sum_{i}\left(y_{i}-\bar{y}_{i}\right)^{2}}}
\end{gathered}
$$

Where $k$ is number of feature. $r_{k c}$ and $r_{k k}$ are linear correlation coefficient between feature and class and between feature and feature, respectively.

However, CFS algorithm cannot define redundant feature.

\section{2) Fast Correlation Based Filter (FCBF) Algorithm}

FCBF algorithm [10] is a algorithm that can analyze irrelevant features and redundant features using symmetrical uncertainty $(\mathrm{SU})$.

$$
\begin{gathered}
S U=2\left[\frac{I G(X \mid Y)}{H(X) H(Y)}\right] \\
I G(X \mid Y)=H(X)-H(X \mid Y) \\
H(X)=-\sum_{i} P\left(x_{i}\right) \log _{2} P\left(x_{i}\right)
\end{gathered}
$$

Where $\operatorname{IG}(\mathrm{X} \mid \mathrm{Y})$ is the information gain of feature $\mathrm{X}$ given condition of feature $\mathrm{Y} . \mathrm{H}(\mathrm{X})$ and $\mathrm{H}(\mathrm{Y})$ are the entropy of feature $\mathrm{X}$ and $\mathrm{Y}$, respectively. 
Irrelevant feature analysis will consider SU that it is the modified of information gain. SU has rank between 0 and 1 . If SU between feature and class equal to 1 , it mean that this feature is completely related to that class. On the other hand, if SU between feature and class equal to 0 , this feature is irrelevant to that class.

A feature $i$ is considered as a redundant feature to feature $j$ if the condition of SU between feature $i$ and feature $j\left(S U_{i, j}\right)$ and $\mathrm{SU}$ between feature $i$ and class $c\left(S U_{i, c}\right)$ is according to equation 6.

$$
S U_{j, c} \geq S U_{i, c} \text { and } S U_{i, j} \geq S U_{i, c}
$$

\section{3) Max-Relevant, Min-Redundant (mRMR) Algorithm}

mRMR algorithm [11] find optimal features that related to class with low redundancy. This algorithm consists of maxrelevant analysis in equation 7 and min-redundant analysis in equation 8 .

$$
\begin{aligned}
& \max D(S, c), D=\frac{1}{|S|} \sum_{x_{i} \in S} I\left(x_{i} ; c\right) \\
& \min R(S), R=\frac{1}{|S|^{2}} \sum_{x_{i}, x_{j} \in S} I\left(x_{i}, x_{j}\right)
\end{aligned}
$$

Where $\mathrm{D}$ is dependency of features. $\mathrm{R}$ is redundancy of features. $|\mathrm{S}|$ is number of feature set. $I\left(x_{i} ; c\right)$ and $I\left(x_{i} ; x_{j}\right)$ are information gain between feature and class and between feature and feature, respectively. This algorithm is calculated from find optimal features that has max-relevant and min-redundant.

$$
\max \Phi(D, R), \Phi=D-R
$$

Although, the output of this algorithm is optimal feature, it cannot identify both irrelevant features and redundant features.

\section{B. Causal Feature Selection}

\section{1) PC Algorithm}

PC algorithm [12] uses Bayesian network which is constraint-based type. This algorithm consists of two processes; edge detection and edge orientation. Edge detection uses conditional independent of all nodes (features) to identify skeleton of edge (related line) between features and features. After that, edge orientation process used d-separation [7] to determine direction of all of edge as shown in figure 4.

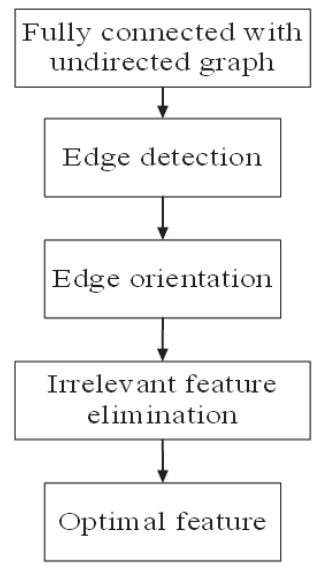

Fig. 4. PC algorithm

2) Parent-Children Based for Causal Redundant Feature Identification (PCRF) Algorithm

This paper proposes PCRF algorithm that uses parent and children relation to find causal graph which is based on IAMB algorithm [13] as shown in figure 5. First, the class is identified and remove irrelevant features that does not related (disconnected) with the class (component I in figure 1) by using Bayesian Networks. After that, information gain in equation 10 is used to find optimal feature (component III and IV in figure 1). Finally, redundant feature can be identified (component II in figure 1) in causal feature selection by removing irrelevant (component I in figure 1) and optimal features (component III+IV in figure 1) from original features. The pseudo code of PCRF algorithm is shown in figure 6 .

$$
I(x ; c)=\iint p(x, c) \log \frac{p(x, c)}{p(x) p(c)} d x d c
$$

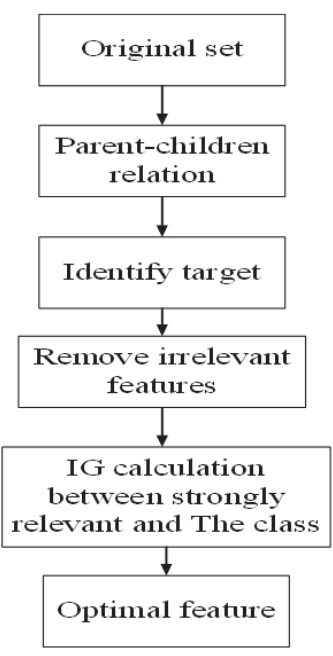

Fig. 5. PCRF algorithm 


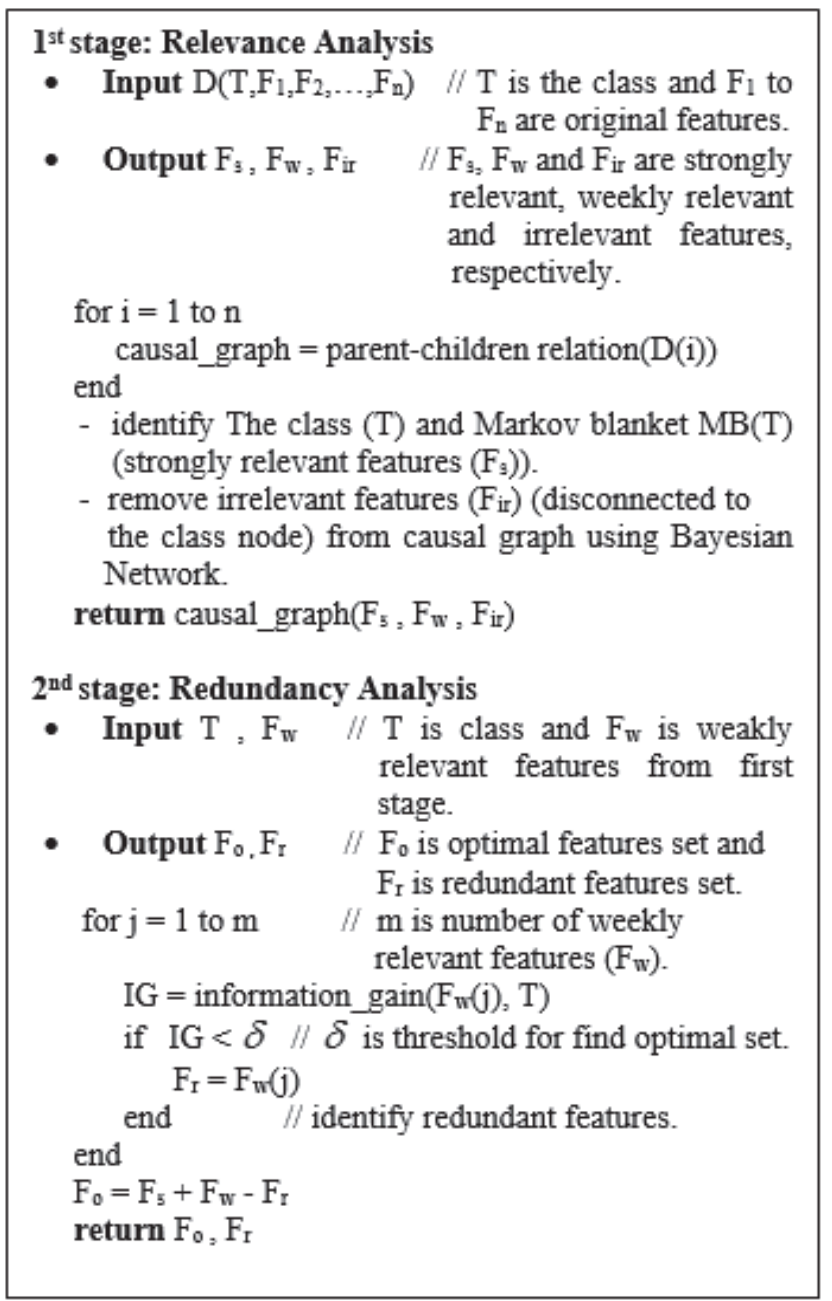

Fig. 6. Pseudo code of PCRF algorithm

\section{EXPERIMENTAL SETUP}

This experiment uses four medical datasets from UCI machine learning repository [14]; Heart disease (HEART), HEPATITIS, PARKINSON and Lung cancer (LUNG) and a dataset from Causality challenge [15]; LUCAS as shown detail in table 1.

TABLE I. Detail of Dataset in EXPeriment

\begin{tabular}{|l|c|c|c|}
\hline \multicolumn{1}{|c|}{ Dataset } & Sample & Feature & Class \\
\hline HEART & 303 & 13 & 2 \\
\hline HEPATITIS & 155 & 19 & 2 \\
\hline PARKINSON & 195 & 22 & 2 \\
\hline LUNG & 32 & 56 & 3 \\
\hline LUCAS & 2,000 & 11 & 2 \\
\hline
\end{tabular}

This experiment measures the accuracy using well-known classifier. Naïve Bayes Classifier (NBC), K-Nearest Neighbor $(\mathrm{KNN}) \mathrm{k}=5$ and Decision Tree (DT). The experiment uses 10fold cross validation with 10 repetition times.

\section{EXPERIMENTAL RESULT}

TABLE II. RESUlt OF FEATURE Reduction

\begin{tabular}{|c|c|c|c|c|c|c|c|}
\hline 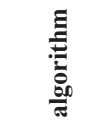 & 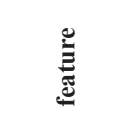 & 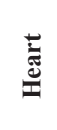 & 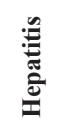 & 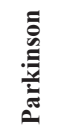 & لِ & $\underbrace{n}_{0}$ & 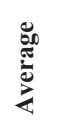 \\
\hline \multirow{3}{*}{ CFS } & Irrelevant & 4 & 4 & 2 & 47 & 2 & 11.8 \\
\hline & Redundant & N/A & N/A & N/A & N/A & N/A & N/A \\
\hline & Selected & 9 & 15 & 20 & 9 & 9 & 12.4 \\
\hline \multirow{3}{*}{ FCBF } & Irrelevant & 7 & 7 & 2 & 20 & 4 & 8.0 \\
\hline & Redundant & 1 & 4 & 14 & 27 & 2 & 9.6 \\
\hline & Selected & 5 & 8 & 6 & 9 & 5 & 6.6 \\
\hline \multirow{3}{*}{ mRMR } & Irrelevant & N/A & N/A & N/A & N/A & N/A & N/A \\
\hline & Redundant & N/A & N/A & N/A & N/A & N/A & N/A \\
\hline & Selected & 5 & 12 & 12 & 6 & 5 & 8.0 \\
\hline \multirow{3}{*}{$\mathrm{PC}$} & Irrelevant & 3 & 1 & 0 & 0 & 2 & 1.2 \\
\hline & Redundant & N/A & N/A & N/A & N/A & N/A & N/A \\
\hline & Selected & 10 & 18 & 22 & 56 & 9 & 23.0 \\
\hline \multirow{3}{*}{ PCRF } & Irrelevant & 2 & 1 & 1 & 16 & 1 & 4.2 \\
\hline & Redundant & 2 & 3 & 0 & 7 & 3 & 3.0 \\
\hline & Selected & 9 & 15 & 21 & 33 & 7 & 17.0 \\
\hline
\end{tabular}

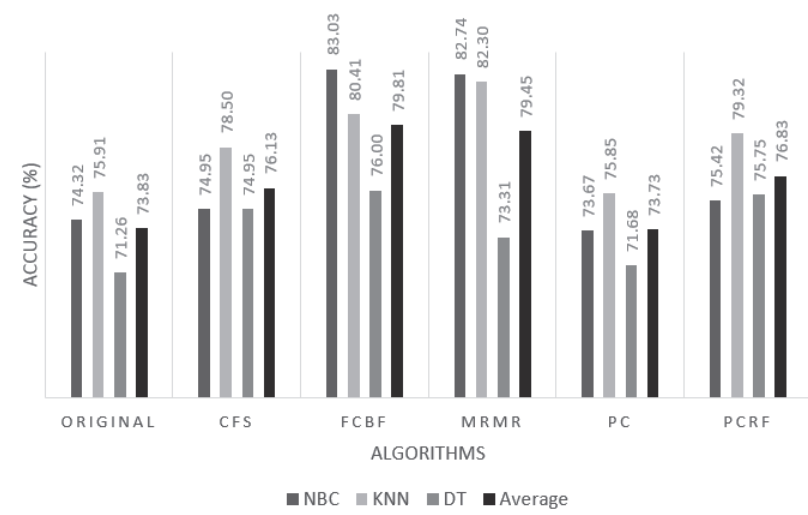

Fig. 7. Average of Accuracy of Classifier from 5 Datasets

Table 2 shows the result of feature reduction. According to the results, FCBF and $\mathrm{mRMR}$ algorithm can reduce more features than others feature selection algorithm. FCBF algorithm can reduce number of average features to $6.6,8$ features for mRMR algorithm and 12.4 features for CFS algorithm while 
PCRF and PC algorithm have number of features 17 and 23, respectively.

Figure 7 presents the result of accuracy of classification. From the graphs, FCBF algorithm and mRMR algorithm provide higher average of accuracy of classifier than other algorithms. FCBF algorithm provides average accuracy $79.81 \%$, $79.45 \%$ for mRMR algorithm and $76.13 \%$ for CFS algorithm while PCRF and PC algorithm provide average accuracy $76.83 \%$ and $73.73 \%$, respectively.

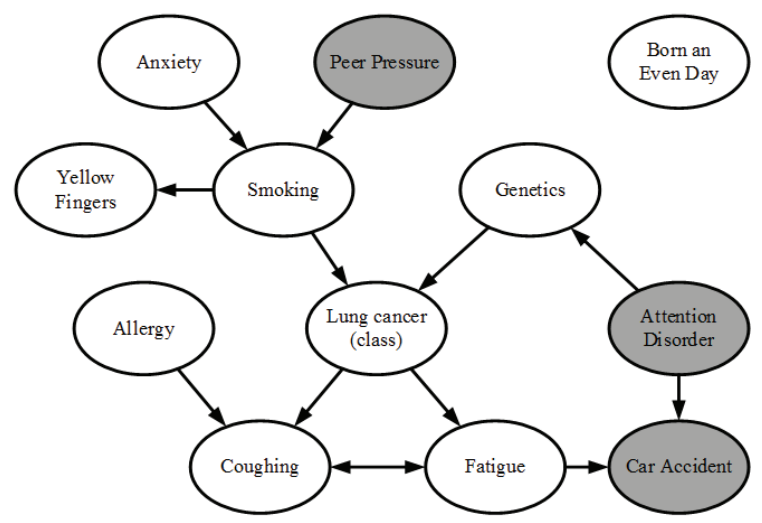

Fig. 8. Causal graph of LUCAS dataset using PCRF algorithm

Figure 8 presents a sample causal graph result of LUCAS dataset using PCRF algorithm. From this figure, PCRF algorithm can identify causal relation between features, irrelevant feature (disconnected node) and redundant features (gray nodes).

\section{DISCUSSION}

From experimental results, FCBF and mRMR algorithms have the average of accuracy of classification higher than other feature selections. Both algorithms also reduce number of feature in data more than other algorithm because it can remove both irrelevant and redundant features while CFS and PC algorithm cannot remove redundant features higher number of selected features.

PCRF algorithm has slightly lower average of accuracy of classification than CFS algorithm but it has higher accuracy than PC algorithm because PCRF algorithm can remove both irrelevant features and redundant features.

Although PCRF algorithm can identify and remove redundant features in causal feature selection, it cannot use with very high dimensional dataset because its complexity. It has to calculate parent-children relationship from every features.

\section{CONCLUSION}

This paper presents redundant feature identification and redundancy analysis for causal feature selection using parentchildren based for redundant feature identification (PCRF) algorithm. From experimental result, PCRF algorithm can identify redundant features and eliminate both irrelevant and redundant features in causal feature selection but has lower accuracy of classification than correlation. However, PCRF algorithm can present causal relation between features and the class. Future work will identify and remove irrelevant and redundant feature by using statistic based for faster calculation in high dimensional data.

\section{REFERENCES}

[1] M. Verleysen and D. François, "The curse of dimensionality in data mining and time series prediction," in Computational Intelligence and Bioinspired Systems, Springer, 2005, pp. 758 770.

[2] L. Yu and H. Liu, "Efficient feature selection via analysis of relevance and redundancy," The Journal of Machine Learning Research, vol. 5, pp. 1205-1224, 2004.

[3] Z. M. Hira and D. F. Gillies, "A review of feature selection and feature extraction methods applied on microarray data," 2015.

[4] H. Liu and L. Yu, "Toward integrating feature selection algorithms for classification and clustering," Knowledge and Data Engineering, IEEE Transactions on, vol. 17, no. 4, pp. 491502,2005

[5] Y. Saeys, I. Inza, and P. Larrañaga, "A review of feature selection techniques in bioinformatics," bioinformatics, vol. 23, no. 19, pp. 2507-2517, 2007.

[6] C. F. Aliferis, I. Tsamardinos, and A. Statnikov, "HITON: a novel Markov Blanket algorithm for optimal variable selection," in AMIA Annual Symposium Proceedings, 2003, vol. 2003, p. 21.

[7] I. Guyon, C. Aliferis, and A. Elisseeff, "Causal feature selection," Computational methods of feature selection, pp. 63-86, 2007.

[8] I. Ben-Gal, "Bayesian networks," Encyclopedia of statistics in quality and reliability, 2007.

[9] M. A. Hall, "Correlation-based feature selection for machine learning," The University of Waikato, 1999.

[10] L. Yu and H. Liu, "Feature selection for high-dimensional data: A fast correlation-based filter solution," in ICML, 2003, vol. 3, pp. 856-863.

[11] H. Peng, F. Long, and C. Ding, "Feature selection based on mutual information criteria of max-dependency, max-relevance, and min-redundancy," Pattern Analysis and Machine Intelligence, IEEE Transactions on, vol. 27, no. 8, pp. 1226-1238, 2005.

[12] P. Spirtes, C. N. Glymour, and R. Scheines, Causation, prediction, and search, vol. 81. MIT press, 2000.

[13] Y. Zhang, Z. Zhang, K. Liu, and G. Qian, "An improved IAMB algorithm for Markov blanket discovery," Journal of Computers, vol. 5, no. 11, pp. 1755-1761, 2010.

[14] A. Asuncion and D. Newman, "UCI machine learning repository," $2007 . \quad$ [Online]. Available: http://www.ics.uci.edu/»mlearn/MLRepository.html

[15] Guyon, I.: Causality Workbench http://www.causality.inf.ethz.ch/home.php
(2008) 